\title{
Optical size effects in thin gold films
}

\author{
S.A. Kovalenko, M.P. Lisitsa \\ Institute of Semiconductor Physics NAS of Ukraine, 41 prospect Nauky, 03650 Kyiv, Ukraine
}

\begin{abstract}
The optical size effects that appear in thickness dependencies of refraction $(n)$ and absorption $(k)$ indexes, Brewster's quasiangle and dielectric constant $\varepsilon_{2}$ in thin gold films are discussed. All of them have oscillating features in the range of thicknesses $20<d<180 \AA$. The errors of determining the optical constants from the photometric data were carefully analyzed.
\end{abstract}

Keywords: thin gold film, refraction and absorption indexes, permittivity, size effects, Brewster's quasiangle.

Paper received 11.09.01; revised manuscript received 23.07.02; accepted for publication 10.12.02.

\section{Determination of $n$ and $k$ using $R, T$ measurements}

The measurement of reflection $R$ and transmission $T$ indexes at the normal fall of light on an investigated sample for determination of thin film optical constants within a broad spectral range is used the most frequently.

The dependence between $R, T$ and optical constants is described by the Murman correlation [1] and formally looks like:

$$
\begin{aligned}
& R=f(n, k), \\
& T=\varphi(n, k) .
\end{aligned}
$$

It is impossible to solve this combined equations relatively $n$ and $k$, therefore the approximate methods for example, described in [2,3] are frequently used, that does not allow to provide high accuracy, in particular, when objects of research are thin films. The graphic methods give the worse results. As it was informed in works [4,5], using such methods for superthin metal films is completely excluded, as at the $d / \lambda<0.05$ correlation it is not possible to determine $n$ and $k$ at all, because curves, which solutions find on the point of intersection, are not crossed.

At first we shall consider a method that consists in definition of coordinates point of intersection curves of equal reflection and transmission to analyse the real picture and to determine possible errors using photometric data for calculation $n$ and $k$.

Let's consider the absorption coefficient $k$ as already known. Each equation of the system (1) under such con- dition turns into the equations with one unknown quantity:

$$
\begin{aligned}
& n_{R}=\psi(k), \\
& n_{T}=\chi(k) .
\end{aligned}
$$

If these are depicted graphically, we shall have two curves with two common points with coordinates $n_{1}, k_{1}$ and $n_{2}, k_{2}$. It is easy to establish, which of them fit true values to required parameters, using the conditions:

$$
\begin{aligned}
& n>k, \text { at } \lambda<\lambda_{p} \\
& n<k, \text { at } \lambda>\lambda_{p},
\end{aligned}
$$

where $\lambda_{p}$ - wavelength of bulk electronic plasma resonance. In case of metals, it suits an ultra-violet region of spectrum. Specifically, for gold $\lambda_{p}=385 \mathrm{~nm}$.

Finding roots of the equations (2) by using the computer consists of three stages.

In the first one, we find an interval $k_{\min }<k<k_{\max }$ within set $k$ values for which determined $n$ values exist.

In the second one with rather big step $\Delta k \approx 0.2$, we find interval $\Delta k$ where the $n_{R}(k)-n_{T}(k)$ difference changes sign, that is the curves (2) cross.

At the third stage, we find the exact values of $\Delta k, n_{1}$ and $k_{1}$ using the half-division method.

In practice, one can meet cases when $R$ and $T$ measured with $1 \%$ errors, while the points of intersection of the curves (2) do not exist. It is caused not only by errors of determining, $R$ and $T$, but their presence when measuring a thin layer thickness $d$. Due to complexity of func- 


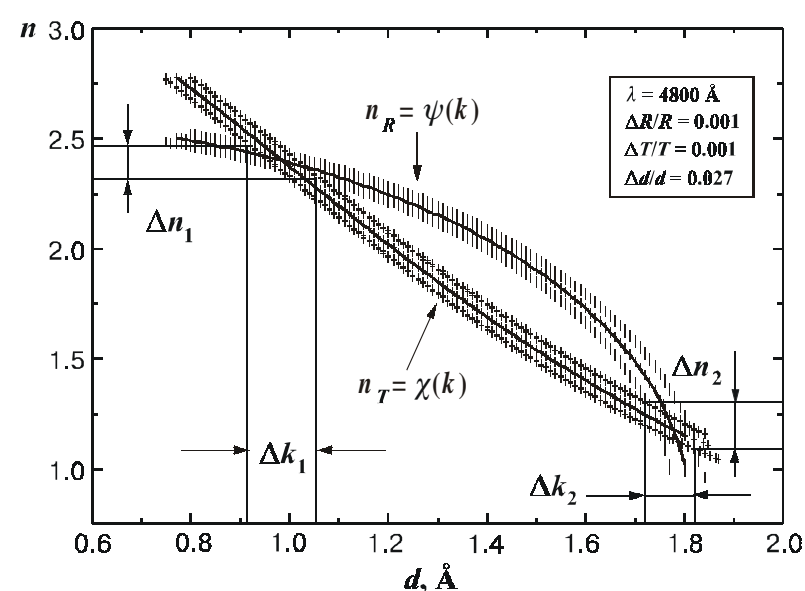

Fig. 1. Errors in determining the optical constants $n$ and $k$ of gold film with thickness $d=185 \AA, \lambda=4800 \AA$.

tional dependences $n(R, T, d)$ and $k(R, T, d)$ with known errors $\Delta R, \Delta T, \Delta d$, it is practically impossible to define errors $\Delta n$ and $\Delta k$ in an explicit form. Therefore, instead of obtaining curves of equal reflection and transmission we have constructed bands of the same types, having calculated with this purpose $n$ and $k$ using extreme values $R$, $T$ and $d$, that is $n(R \pm \Delta R, T \pm \Delta T, d \pm \Delta d)$ and $k(R \pm \Delta R$, $T \pm \Delta T, d \pm \Delta d$ ). Section of crossing these bands will give bounds where roots of the system (2) change.

Let's consider specifical cases starting with the thickest gold layer from those investigated in this paper. It is fit the diagram shown in Fig. 1, in the insert of which relative errors of measuring $R, T$ and thickness $d$ of the layer are given. The extreme and average values of $n$ and $k$ are determined from with the width of bands in intersection ranges. The latter are accordingly equal to 1.18 and 1.78. For the ratio $d / \lambda=0.038$, the possible value ranges of optical constants are as follows $1.07<n<1.30$, $1.71<k<1.82$. The relative error for $n$ makes $19 \%$, for $k-6.1 \%$.

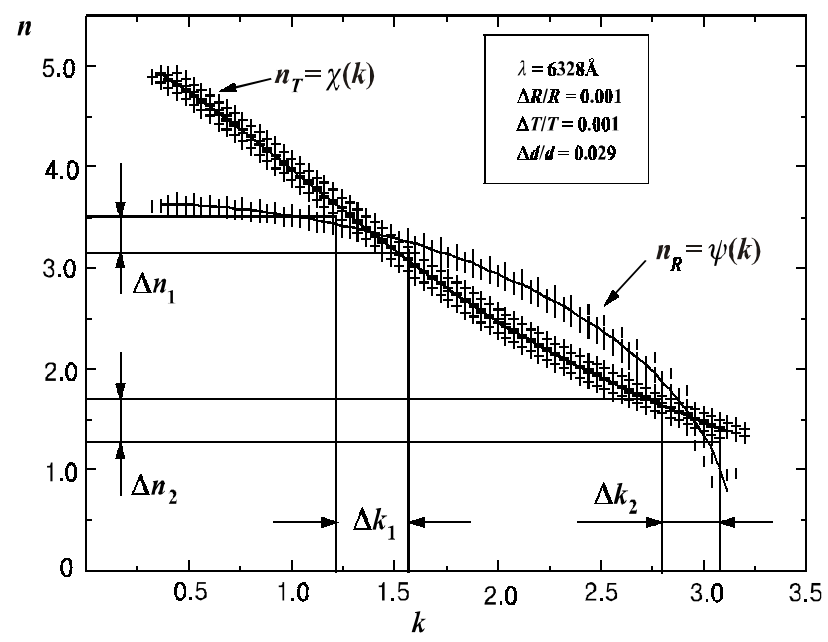

Fig. 2. Errors in determining the optical constants $n$ and $k$ of gold film with thickness $d=85 \AA, \lambda=6328 \AA$.
The errors became greater (Fig. 2) at the same accuracy of experimental measurements of $R, T$ and a little smaller one when determining the thickness of $85 \AA$ gold film using wavelength $\lambda=6328 \AA$. Average values: $n=1.5$, $k=2.95$; intervals of possible values: $1.27<n<1.69$, $2.80<k<3.07 ; \Delta n / n=28 \% ; \Delta k / k=9 \% ; d / \lambda=0.01$.

At last, in Fig. 3 shown is the similar plot of error determination in the case of the thinnest investigated films if keeping the previous accuracy of $R, T$ measurements and of too much smaller when measuring their thickness is given. The average values of $n$ and $k$ are accordingly equal 0.49 and 2.98 . Intervals, in which there are their true values wider in general than in previous cases, namely: $0.35<n<0.68 ; 2.53<k<3.34$. Ratio error for $n$ and $k$ are accordingly equal $27 \%$ and $67 \%$.

In all three cases, according to the conditions (3), true values of $n$ and $k$ fit to those present in the range of error band intersection at the big $k$ values (the right edge of each diagram). They testify that with reduction of $d$, more precisely of the ratio $d / \lambda$, decreased is also the accuracy of determining the optical constants. It occurs due to the big inaccuracy of measurements of thickness and specificity of the equations (2). Even increase of accuracy of measurement of factors $R$ and $T$ up to $0.1 \%$ do not allow to determine $\mathrm{n}$ and $k$ for a film with thickness $20 \AA$ with an error smaller than $20 \%$ as illustrated by these plots.

\section{Thickness dependences of optical parameters. $n$ and $\boldsymbol{k}$ parameters}

In the paper [6], it is developed the reliable, as authors consider, method of determination of optical constants that supposedly allows to solve correctly a return photometric problem and always find the valid solutions. As they with the silver films having the thickness $35-150 \AA$ investigated in a visible spectrum the, $d / \lambda$ ratio changed within the bounds that to some extent allowed to hope for obtaining $n$ and $k$ values close to the real ones. However,

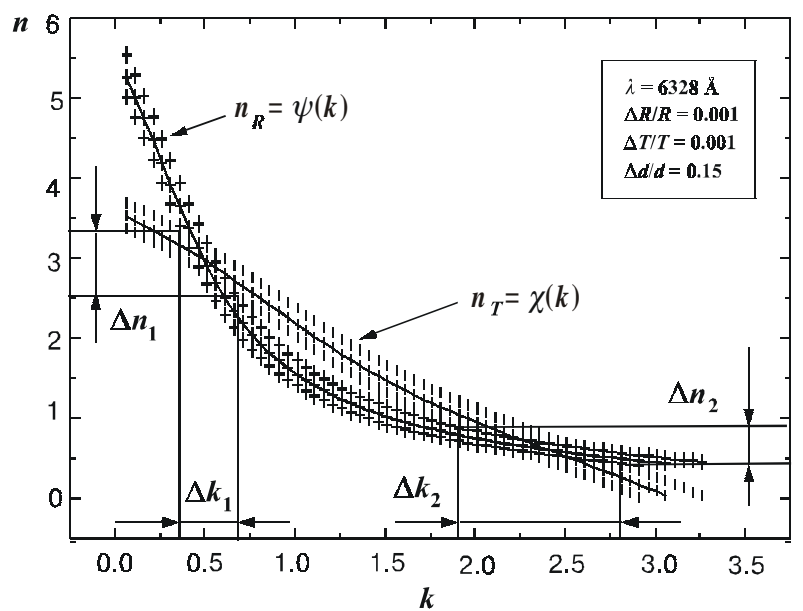

Fig. 3. Errors in determining the optical constants $n$ and $k$ of gold film with thickness $d=20 \AA, \lambda=6328 \AA$. 


\section{S.A. Kovalenko, M.P. Lisitsa: Optical size effects in thin gold films}

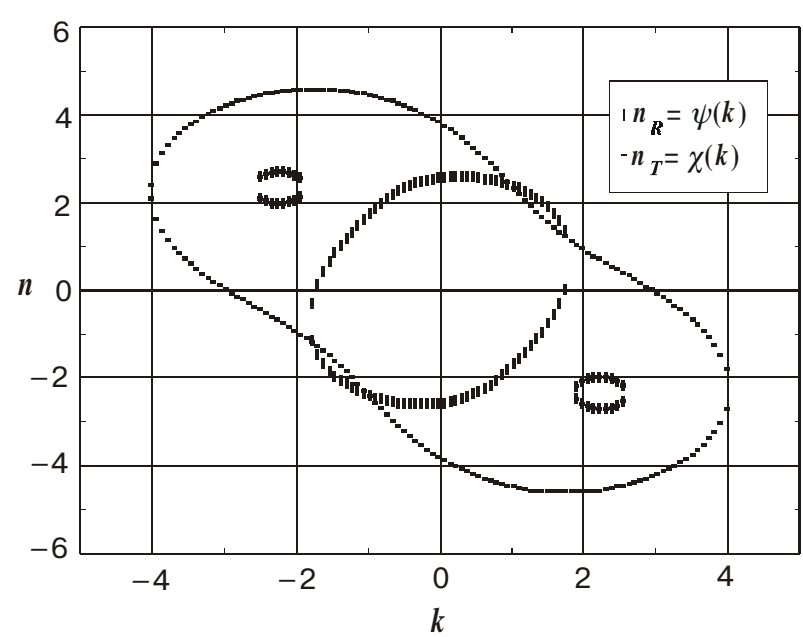

Fig. 4. Set of all possible solutions for a film $d=185 \AA, \lambda=4800 \AA$.

for thin bismuth films, within the thickness range $300<d<1000 \AA$ investigated in infra-red spectral region $(25<\lambda<125 \mathrm{~mm})$, the ratio $d / \lambda$ appears too small to provide necessary accuracy requirements.

The thickness of gold films used by us and visible spectrum, in which researches were carried out, fit to conditions, using of which allows to speak about legitimacy of applying the data obtained in the given photometric measurements at the normal light incidence for determination of optical constants, but mistakes, when calculating of $n$ and $k$ on their basis, were too great. It has forced us to search for an other method that would provide a smaller error.

One more fact has forced us to search for another method of determination of optical constants. In Fig. 4 given is the full set of solutions that fits to Fig. 1. As shown in this diagram, there are only two roots that would fit to the conditions $n>0, k>0$ and it is sufficient to use condition (3) for a choice of the true solution. But we can have even four roots (Fig. 5), as it was written by Ables

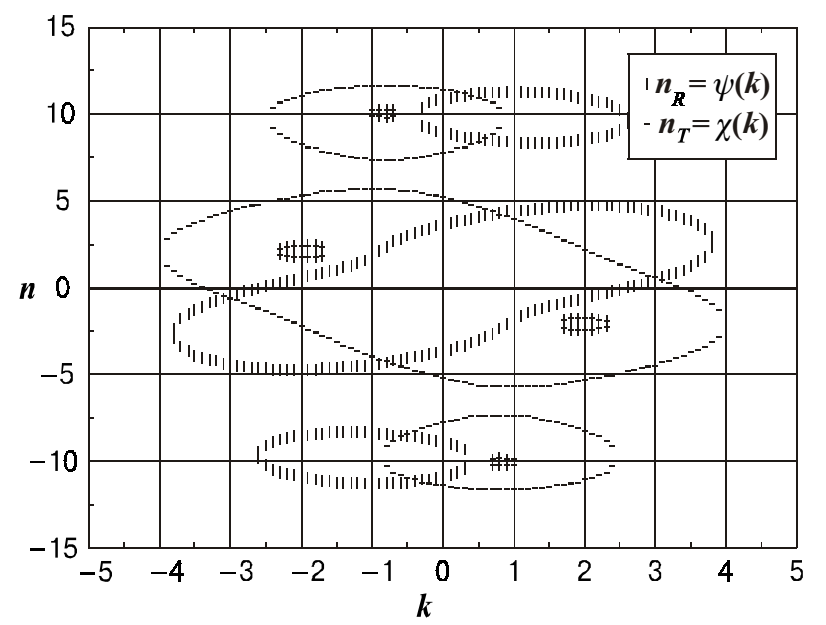

Fig. 5. Solution set of system (1) for data of paper [7], $d=250 \AA$, $\lambda=5000 \AA, n_{s}=1.52, R_{\text {exp }}=0.6, T_{\text {exp }}=0.2$.

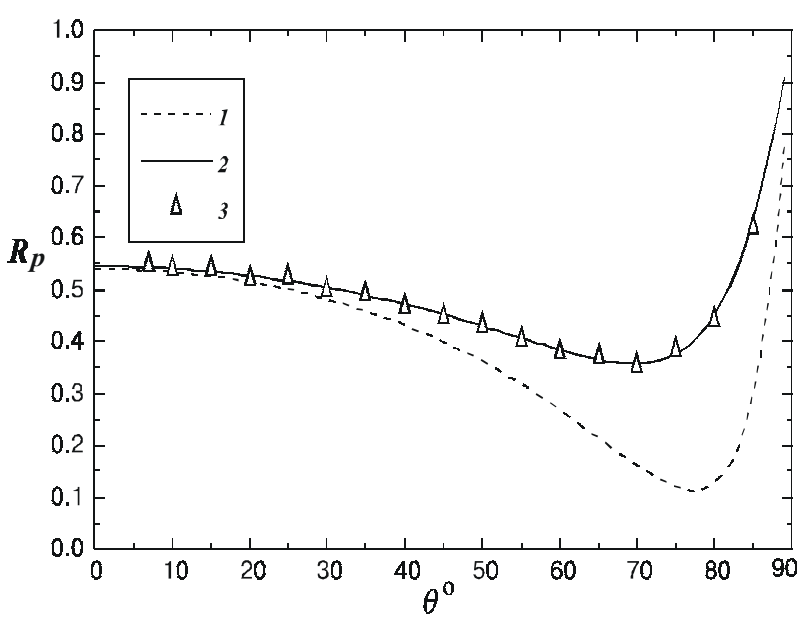

Fig. 6. Angular dependence of power reflection factor $p$-polarization for a gold film $d=185 \AA, \lambda=6328 \AA$ : $l$ - calculation for $n_{1}=4.38, k_{1}=0.21 ; 2-$ calculation for $n_{1}=0.18, k_{1}=3.65 ; 3-$ experimental data.

[7]. In that case, the method of measuring reflection factors at oblique incidence of polarized light relieves. The angular dependences of the reflection factor for the $p$ polarization are different in all pairs of $n$ and $k$ solutions that give identical $R$ and $T$ at the normal incedence, as Fig. 5 illustrates.

According to our experimental data for the case given in Fig. 1, the angular dependences $R_{p}$ are depicted in Fig. 6.

Thus, shown is the possibility to use a condition (3) when chosing the true solution, and it is even better, the plots given on Figs 5, 6. Besides, as is shown in [8], the calculations that have been carried out according to angular measurements have the greater accuracy of determination $n$ and $k$. It is explained that $R_{s}$ and $R_{p}$ depend on optical constants more strongly than $R$ and $T$ at normal incidence.

So, considering all facts described above, the standard measurement method of angular dependences of reflection factors in two mutually perpendicular polarizations, that is $R_{s}$ and $R_{p}$, was chosen among different variants.

With this purpose, the installation, the optical schematic of which is given in Fig. 7, was collected. The laser LG-79 served in it as the light source 1, it was possible to

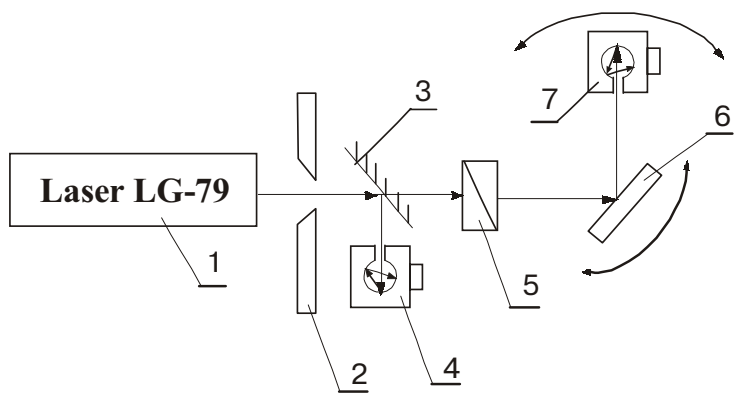

Fig. 7. The optical schematic of setting for angular measurements $R_{p}$ and $R_{s}$. 


\section{S.A. Kovalenko, M.P. Lisitsa: Optical size effects in thin gold films}

limit diameter of beam cone by the diaphragm 2. The certain part of beams was removed by the beam divider 3 on a photodetector 4 that allowed to determine intensity of laser radiation. Polarizer 5 the necessary component of beams falling onto the sample 6 . The detector 7 measured intensity of the beams reflected by it.

It was important to exclude the beams reflected by a free surface of a substrate in the measurements carried out. It was easily performed by a choice of glass plates with a significant thickness. The minimal angle, at which it was yet possible to divide the first and second beams, made $6^{\circ}$. The maximal one, at which the laser bunch did not fall outside the limits of the sample, made not less than $87^{\circ}$. The step of measurement was chosen as one arc degree.

The optical constants $n$ and $k$ were calculated from forty used angles of incidence, and their average value is found. Besides, the averaging was also carried out using the sample area, as with increasing angle of incidence the surface covered with light was increased, too. The influence of relief defects was partly excluded by it.

To calculate angular dependences of $R_{p}$ and $R_{s}$, the formulae in matrix representation [9] were used. They have the identical degree of accuracy with the Murman formulae.

During this work, it appeared that more convenient is to use an iterative technique of computer processing of experimental data using the least-square method that smoothed experimental curves $R_{p}$ and $R_{s}$ and simultaneously allowed to calculate $n$ and $k$.

The thickness dependences of index of refraction and absorption of the gold films received by the way described above, are given in Fig. 8. The sizes of rectangular points define size of an error.

In particular, careful measurements were carried out on the films of small thickness (20-65 $\AA$ ), and errors were determined separately for each case. Their values are shown in Tab. 1.

As long as according to data [10] the length of electron free path for gold is equal $\approx 400 \AA$, the sharp increase of an index of refraction begins at thickness approximately three times smaller. In our opinion, it is manifestation of classical dimensional effect. According to the simple theory of optical constants of metals the contribution of free carriers to the value of a refraction index is negative. And with reduction of film thickness down to the specified value of electrons scattering on film borders becomes essential, therefore their influence decreases,

Table 1. Errors of determining $\boldsymbol{n}$ and $\boldsymbol{k}$ of gold films with a different thickness

\begin{tabular}{ccc}
\hline \hline$d, \AA$ & $\Delta n / n, \%$ & $\Delta k / k, \%$ \\
\hline 20 & 0,5 & 15 \\
\hline 35 & 1,3 & 8 \\
\hline 40 & 2,4 & 7 \\
\hline 50 & 1,8 & 3,6 \\
\hline 60 & 1,2 & 3,8 \\
\hline \hline
\end{tabular}

SQO, 5(3), 2002

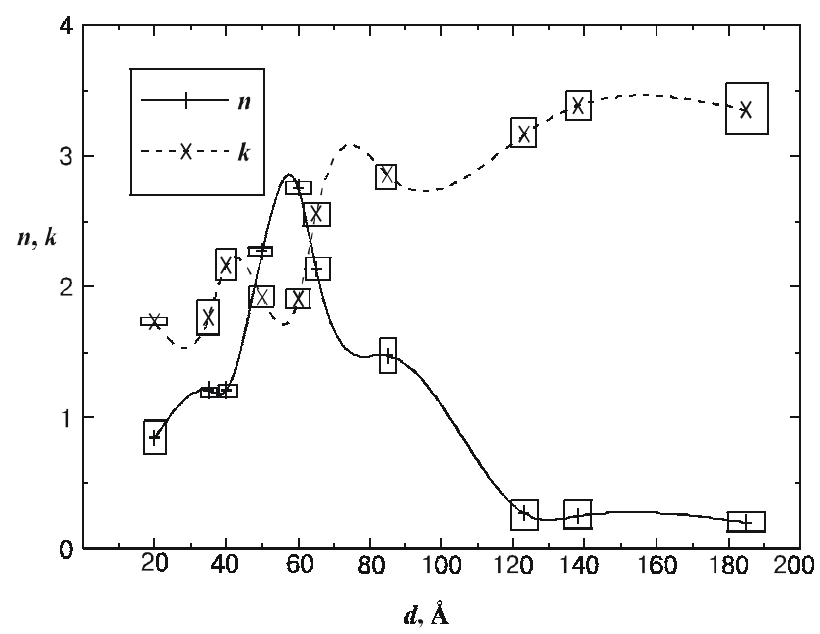

Fig. 8. Thickness dependences of index of refraction $n$ and absorption $k$ of gold films, $(\lambda=6328 \AA)$.

and the refraction index grows reaching the principal maximum at $d \approx 60 \AA$ that is almost 7 times smaller than the electron free path length. Further, there is a decrease in the refraction index due to reduction of material film density, which is proportional to optical density. At the thickness close to zero it tends to unity - the index of air refraction.

The best accuracy of determinition of optical constants that is provided by measurements of $R_{p}$ and $R_{s}$ angular dependences allows to observe oscillations of $n$ and $k$ (Fig. 8), which belong to quantum dimensional effects, as optical properties of substance change in discrete steps at energy and quasi-momentum quantization.

This phenomenon is shown in thin films and inversion layers at thickness about length of the de Broglie wave for free carriers:

$d \approx \lambda_{D}=\frac{h}{p}=\frac{2 \pi \hbar}{\sqrt{2 m^{*} E}}$

where $h$ - Planck's constant, $p=m^{*} v$ - an electron moment, $m^{*}$ - its effective mass, $v$-speed of thermal motion, $\hbar=h / 2 \pi, E-$ energy.

According to formula (4) estimations give for gold the value $\lambda_{D} \approx 6 \AA$ that is close to a constant of crystal lattice of a massive sample of this material.

The classical example of quantum-dimensional phenomena is thickness dependences of an imaginary part of complex permittivity of nickel, tungsten and titanium films, investigated by L. A. Kuzik with co-authors [11]. The authors observe the oscillations with 5-7 maxima and minima and the period about half of the de Broglie wave in the interval of thicknesses 2 to $18 \AA$. Unfortunately, low accuracy of a photometric method did not allow them to receive the appropriate dependences for $n$ and $k$.

In our case oscillations occur at much greater thickness and the period does not coincide with size of the de Broglie wave. But similar correlation is also present in [12], authors of which investigated dependence of an aluminium film transparency on the thickness, and in [13] it is proved theoretically. Moreover, the presence of di- 


\section{S.A. Kovalenko, M.P. Lisitsa: Optical size effects in thin gold films}

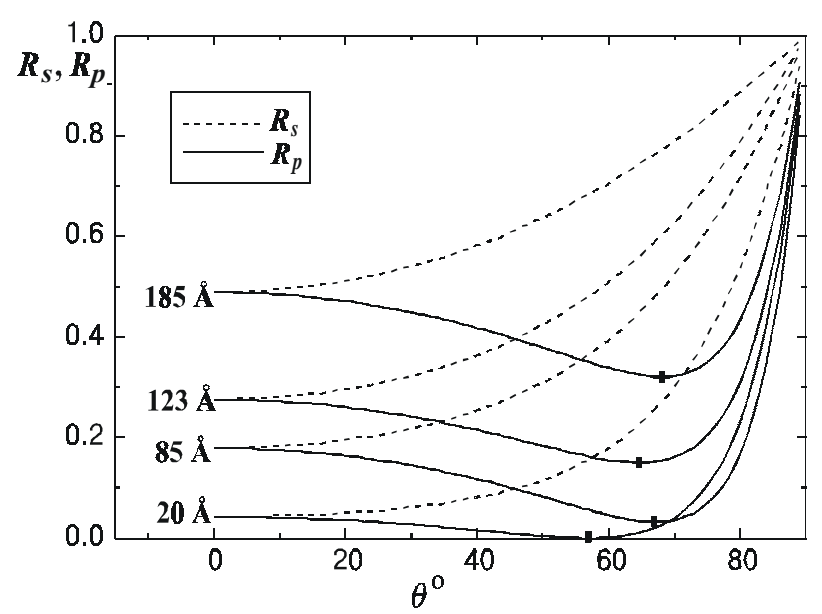

Fig. 9. Angular dependences $R_{p}$ and $R_{s}$ for gold films on glass substrate $\left(n_{s}=1.512, \lambda=6328 \AA\right)$.

mensional quantization in gold films is proved by dimensional oscillations considered in the following section.

\section{Brewster's angle, $R_{p}, R_{s}$ and $\operatorname{Im} \varepsilon$}

Angular dependences of Frenel's factors $R_{p}$ and $R_{s}$ for gold films of four thicknesses are shown in Fig. 9. It shows that values of Brewster's quasiangle and Fresnel's factors are functions of a film thickness, the former having oscillation character. The latter, as a matter of fact, is monotonous; the more film thickness, the greater are values of $R_{p}$ and $R_{s}$. Indeed, the thinnest film $(d=20 \AA)$ has the greater values than received for a film with thickness $85 \AA$ A. This anomaly may be caused by presence of surface waves, energy of which also may represent itself as a function of thickness. Being spread along a surface of a layer, such waves are not registered by the detector, but take away a part of energy from the reflected light beam.

The oscillation character of Brewster's quasiangle for gold films, put on a glass lining, and also free films represents by Fig. 10. The first curve has two dim maxima,

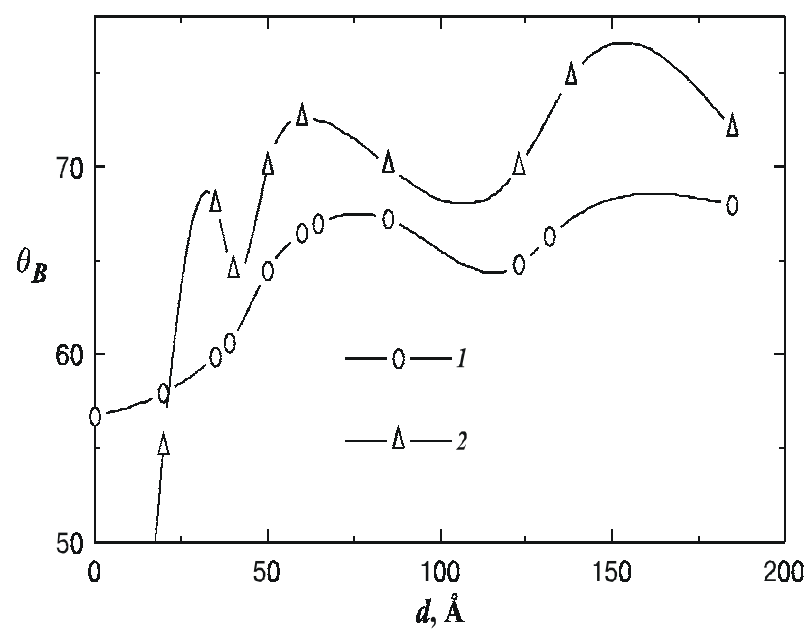

Fig. 10. Dependence of Brewster's quasiangle on thickness of a gold film, $\lambda=6328 \AA$ : $I$ - for a film put on glass, 2 - for a free film.

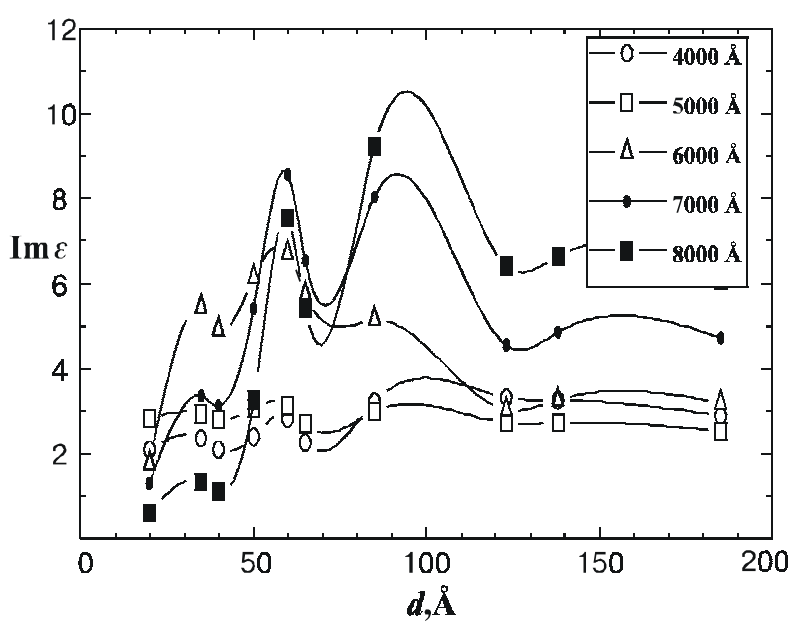

Fig. 11. Dependence of an imaginary part of complex permittivity $\varepsilon_{2}$ on thickness of gold film.

and the second - one. The last one covers an interval of the lowest used values of thickness. As long as it is technologically impossible to obtain free films with tens $\AA$ thickness, the second curve is a result of calculations using the received values of $n$ and $k$.

It is interesting to look after changes of imaginary component of a permittivity $\varepsilon_{2}$ with thickness, after all it is a function itself both of the refraction index and absorption index, therefore it should have the oscillation character similar to found for $n$ and $k$.

To carry out necessary calculations, we shall use the following formula [11]:

$\varepsilon_{2}=\operatorname{Im} \varepsilon \cong$

$\cong \frac{T_{0}-T}{T_{0}}\left\{4 \pi v d\left[\frac{1}{\sqrt{\varepsilon_{3}}+1}+\frac{2 R \sqrt{\varepsilon_{3}}}{\left(\varepsilon_{3}-1\right)\left(1-R^{2}\right)}\right]\right\}^{-1}$

where $T, T_{0}$ are factors of transmission of a substrate with a gold layer and without it, accordingly; $v$ - frequency; $d$-thickness of a layer; $R$ - factor of reflection by a free surface of the substrate with the permittivity $\varepsilon_{3}$.

$\varepsilon_{3}$ and $R$ are determined according to the known refraction index of the used glass substrate, and $T$ value we find using direct spectral measurements.

The results of calculations are submitted in Fig. 11. They are indicative of the presence of $\varepsilon_{2}$ oscillations for all lengths of waves in the visible spectrum. It is typical that the more the amplitude of oscillations the more length of a wave, and the period coincides with the period of oscillations submitted in Figs 10 and 8.

\section{Conclusions}

1. The span of errors is established when determining optical constants of thin gold films using the experimental measurements of transmission $T$ and reflection $R$ factors in the case of normal beam incidence onto a sample. It has been shown that for films with a thickness less $80 \AA$ 


\section{S.A. Kovalenko, M.P. Lisitsa: Optical size effects in thin gold films}

the method does not provide obtaining the reliable data. Obviously, this conclusion should be taken into consideration while investigating thin films of other substances, too.

2. It was developed a goniometric variant of the photometric method with a laser light source where the measurable values are angular-dependent factors $R_{p}$ and $R_{s}$. The method allows determining $n$ and $k$ with an error of several percents.

3. The thickness dependences of optical constants $n$, $k, \operatorname{Im} \varepsilon$ and Brewster's quasiangle for thin gold films on glass substrate have been investigated. Observed oscillations of these parameters occur due to quantum-dimensional effects.

4. It has been established that change of optical properties of thin gold films becomes essential, since the thickness is approximately three times smaller than lengths of the electron free path in bulk samples of this material. This phenomenon is a manifestation of classical dimensional effect.

\section{Acknowledgements}

This work was partly supported by the International Soros Science Education Program (ISSEP) through the grant \# EPU052023.

\section{References}

1. H. Murmann // Z. Phys., 80, p.161 (1933).

2. Zhuomin M. Zhang. - Optical properties of a slightly absorbing film for oblique incidence // Appl. Optics., 38(1), pp.205207 (1999)
3. J. Thomas, G. Jezequel, I. Pollini, Determinatio of the optical constants of absorbing isotropie materials from multiangle reflectance with polarised synchrotron radiation // JOSA, 5A(3), pp. 344-350 (1988).

4. P. O. Nilsson, Determination of the optical constants from intensity measurements at normal incidence // Appl. Opt., 7(3), pp.435-442 (1968).

5. L. Ward, A. Nag, Theoretical study of sensitivities of some normal incidence methods for measuring the optical constants and thickness of thin films // Brit. J. Appl. Phys., 18(3), pp.277-284 (1967).

6. E.A. Bondar, Yu. A. Kuljupin and N.N. Popovich // The inverse problem of the phenomenological theory of the optical properties of thin films // Thin Solid films, 55, p.201-209 (1978).

7. F. Ables, Optical Proprties of Metallic Films. In: Physics of Thin Films. Eds M. H. Francombe, R. W. Hoffman, 6, p.188, 1971.

8. H. E. Bennett and Jean M. Bennett, Precision Measurements in Thin Film Optics. In: Physics of Thin Films. Eds G. Hass, R. E. Thun, 4, p.72.

9. R. Azzam, N. Bashara., Ellipsometry and polarized light, Moscow, Mir, 1981 (in Russian).

10. D. C. Larson, Size-Dependent Electrical Conduction in Thin Metal Films and Wires. In: Physics of Thin Films. Eds M. H. Francombe, R. W. Hoffman, 6, p.106 (1971).

11. L. A. Kuzik, J. Mattei, F. A. Pudonin, V. A. Yakovlev, Qauantum size effects in optical properties of thin metallic films // Optika i spektroskopiya 83(6), pp. 985-989 (1997) (in Russian).

12. N. E. Alekseevsky, S. I. Vedeneev. Dependences of transparency on thickness of aluminium films. // Pis'ma v ZhETF, 6, pp.865-868, (1967) (in Russian).

13. V. G. Gogan, V. Z. Kresin, The absorption of light in thin films in the presence of quantum size effect. // Fizika tverdogo tela, 11(11), pp. 3230-3235 (1969) (in Russian). 